\title{
A Glossary for Research on Human Crowd Dynamics
}

Consortium for the Physics and Psychology of Human Crowd Dynamics: Juliane Adrian · Martyn Amos · Mitra Baratchi · Mira Beermann · Nikolai Bode · Maik Boltes - Alessandro Corbetta - Guillaume Dezecache · John Drury · Zhijian Fu · Roland Geraerts · Steve Gwynne · Gesine Hofinger · Aoife Hunt · Tinus Kanters · Angelika Kneidl · Krisztina Konya · Gerta Köster · Mira Küpper · Georgios Michalareas · Fergus Neville · Evangelos Ntontis · Stephen Reicher · Enrico Ronchi · Andreas Schadschneider · Armin Seyfried · Alastair Shipman · Anna Sieben · Michael Spearpoint · Gavin Brent Sullivan · Anne Templeton · Federico Toschi · Natalie van der Wal · Frank van Schadewijk · Cornelia von Krüchten · Nanda Wijermans · Zeynep Yücel · Francesco Zanlungo · Iker Zuriguel

Contact details of consortium organizers and glossary editors:

E-mail: nikolai.bode@bristol.ac.uk, a.corbetta@tue.nl, j.drury@sussex.ac.uk, enrico.ronchi@brand.Ith.se, f.toschi@tue.nl, a.seyfried@fz-juelich.de

Received: 17 January 2019 / Last revision received: 27 February 2019 / Accepted: 28 February 2019 DOI: $10.17815 / C D .2019 .19$

\begin{abstract}
This article presents a glossary of terms that are frequently used in research on human crowds. This topic is inherently multidisciplinary as it includes work in and across computer science, engineering, mathematics, physics, psychology and social science, for example. We do not view the glossary presented here as a collection of finalised and formal definitions. Instead, we suggest it is a snapshot of current views and the starting point of an ongoing process that we hope will be useful in providing some guidance on the use of terminology to develop a mutual understanding across disciplines. The glossary was developed collaboratively during a multidisciplinary meeting. We deliberately allow several definitions of terms, to reflect the confluence of disciplines in the field. This also reflects the fact not all contributors necessarily agree with all definitions in this glossary.
\end{abstract} Keywords Agency (collective) · Agent · Behaviour (collective) · Bottleneck · Capacity · 
Clogging, Arching $\cdot$ Cognitive maps $\cdot$ Collective action $\cdot$ Collectivity $\cdot$ Crowd $\cdot$ Emotions (group-based and collective) · Evacuation · Flow · Fundamental diagram · Group · Group coordination · Hand calculation - Lane formation · Model (general, acceleration-based, agent-based, cellular automata, computational, conceptual, deterministic, empirical, formal, hybrid, macroscopic, machine learning, mesoscopic, microscopic, multi-scale, probabilistic or stochastic, velocity-based) - Navigation mesh · Network · Others · Pedestrian · Phenomenon (collective) · Pitch invasion · Queuing · Rituals · Routing · Social appraisal $\cdot$ Social contagion, Social transfer, Social spread $\cdot$ Social identity $\cdot$ Social norm $\cdot$ Social relation $\cdot$ Trajectory $\cdot$ Wayfinding

\section{Glossary}

The glossary is ordered alphabetically. Thus, terms used in some definitions may be defined later in the glossary.

\section{Agency (collective)}

Agency is the ability to act (with freedom)

\section{Agent}

1. Entity making its own decisions. E.g. pedestrians, animals.

2. In the context of pedestrian dynamics, an agent is often a simulated/virtual/modelled pedestrian.

3. Virtual/simulated/modelled pedestrian(s) having characteristics and performing actions or decision making.

4. In programming, an intelligent agent is a computer system capable of flexible autonomous action in some environment, encompassing the following capabilities: Reactive (ability to deal with change); Pro-active (goal-directed behaviour); Social (interact with other actions).

\section{Behaviour (collective)}

1. Behaviour is the response of individuals or groups to internal and external stimuli. Collective behaviours are responses of groups to internal and external stimuli that arise from interactions between group members.

2. Collective behaviour in a group/crowd arises when individuals respond not only to stimuli, but also to others (coordination) leading to the spatial or temporal synchronisation of group/crowd dynamics. In contrast, aggregate behaviour lacks synchronisation across individuals even if interactions between individuals may occur (e.g. to avoid collisions). For example, collective behaviour may occur in a crowd that 
attempts to approach a stimulus together and aggregate behaviour may be observed in a crowd in a shopping centre where individuals separately try to get to different shops.

\section{Bottleneck}

1. Physical obstacle that has a lower throughput capacity than the surrounding environment, such as a narrowing in a corridor. For sufficiently large incoming flows, jamming can occur, resulting in higher densities in front of than behind the bottleneck in the direction of the flow.

2. A physical obstacle/design that can cause a reduction in the flow of a crowd in combination with crowd movement behaviour.

\section{Capacity}

We suggest the definition and related measures depend on the context. Attempted general definition: the maximum number of persons that can be sustained or are allowed to be sustained for a longer period of time performing a certain behaviour in a certain context. Examples include: throughput capacity (maximum stable throughput that can be sustained over a longer period of time per meter width of a crosssection); standing capacity (maximum number of people that can stand in a certain confined area); walking capacity (maximum number of people within an confined space so that walking is still possible).

\section{Clogging, Arching}

When dense crowds push forward towards a narrow exit with high motivation, the bodies of pedestrians can form temporarily stable structures that decrease or block the flow (clogging). The structures often take the shape of concentric semi-circles around the exit, reminiscent of arches over the exit when viewed from above.

\section{Cognitive maps}

A mental representation of reality, often, but not exclusively, relating to physical spaces.

\section{Collective action}

1. A frequently used definition for collective action is a group acting together to improve their situation. However, other authors include all examples of people acting collectively e.g. fans at a concert, supporters at a sporting event etc.

2. Collective actions are collective behaviours that arise from a deliberative decision within groups by means of communication. 


\section{Collectivity}

1. In the context of crowd dynamics: synchronized movement of aggregated individuals/particles, that can be achieved by different means (e.g. physical forces, communication, stimulus-response).

2. Individuals/particles who are considered as a group.

3. The noun is used to refer to the quality of being a group (or group-like).

\section{Crowd}

1. A crowd is an aggregation of people in a physical or non-physical space.

2. A (large) group of people in the same space at the same time. This definition can be further refined into physical crowds (individuals simply in the same place) and psychological crowds (feel as one, share social identity)

3. A (typically large) number of people in one place at the same time. It is possible that a physical crowd contains one or more psychological crowds (e.g. football fans in a transport hub with commuters).

4. Physical crowd: collection of people in the same place comprised of small groups and individuals without a shared social identity (no psychological connectedness with others in the crowd outside of their own subgroups). Psychological crowd: collection of people who share a social identity (the perception that the others in the crowd are ingroup members). In the social identity approach the term partly applies to situations including conflict/novelty. However, many crowds are not in novel situations (e.g. transport hubs, stadia).

\section{Emotions (group-based and collective)}

Group-based emotions are emotions experienced as a function of group membership (e.g. guilt about lack of humanitarian help in a national response to a disaster). Collective-based emotions are group-based positive and negative feelings which are experienced simultaneously and often coordinated with other group members (e.g. joy when your football team scores a goal). The two terms are sometimes used interchangeably, but some scholars argue against this because group-based emotions can be evident merely in the aggregate behaviour of a group or crowd (e.g. the anticipation of fans walking to an important game). Collective emotions, in contrast, occur when most members of a crowd share emotions as a consequence of acting together as a group (e.g. celebrating by singing, engaging in coordinated displays, experiencing their feelings amplified by the sound of the crowd, etc.). Collective emotions can be ascribed only to the whole "psychological group" and its behaviour in a particular context (e.g., protest, event audience, commemoration, etc.). 


\section{Evacuation}

Moving individuals or a crowd to a (safe) location due to a threat or warning or due to safety reasons. There are different types of evacuations:

1. "Invacuation": moving people into buildings for protection against a hurricane, for example

2. "Immediate evacuation": instruct people to leave a building/vehicle immediately

3. "Phased evacuation": evacuation with different phases that cover different locations (e.g. floors in high-rise building) or occupants (e.g. "women and children first")

4. "Non-immediate evacuation": reason to evacuate, but there is quite enough time. A subcategory from this one could be the phased evacuation.

Flow

1. Hydrodynamics relation: flow is the result of the product of average pedestrian density, average speed and and the width of a measurement line or facility.

2. Number of people who pass a measurement line per unit time (can be normalised to people per second per unit length, known as the specific flow).

\section{Fundamental diagram}

The concept of a generalised functional relationship between density and speed or flow. Quantitatively, empirical work has shown that the functional relationships vary across contexts. Qualitative aspects of the relationship may be preserved.

\section{Group}

1. A group of people or items is a number of people or items which are connected or linked for some time by some measure (e.g. spatially or psychologically).

2. In Self-categorization theory (psychological) terms, a group is people who think of themselves as a group (this a theoretical definition).

3. Discussion:

- In crowd modelling a "pedestrian group" is often used to refer to a group of pedestrians, i.e. a group of people moving together. This definition does not specify the nature (or lack) of the social relation between the group members. It would be beneficial to better specify the nature of the group, and it would be useful to agree on sub-categories (e.g. social pedestrian groups defined as groups which have a persistent social relation which goes beyond the current 
crowd dynamical states, such as friends, colleagues and similar; socially interacting pedestrian groups defined as groups whose members are having a social interaction such as conversation while walking together; etc.)

- In some research, groups consist of three or more individuals. In models or simulations, often two individuals (dyads) are described as a group, as the term is used to distinguish single individuals. It could be argued that there should be a consistent definition. Moreover, the question of an upper limit to the size of groups merits consideration. For example, small group research usually agrees that a team or a small group will consist of no more than 7-10 persons. One definition for an upper limit for the size of a groups could be the size for which individuals cannot communicate with every other member of the group anymore.

\section{Group coordination}

Group coordination consists of all the actions and decisions made by the group members to maintain the group. Group coordination is performed by communication with other group members (gaze-following, gestures, vocal interaction).

\section{Hand calculation}

1. Simplified estimation of a certain quantity (e.g. egress time) that can be performed without the aid of simple computational facilities.

2. The determination of a certain quantity or quantities (e.g. evacuation time/s) that typically may be achieved only with the aid of simple computational facilities such as a calculator.

\section{Lane formation}

Self organisation phenomenon occurring in pedestrian crowds that leads to the formation of clusters of pedestrians moving in the same direction. These clusters can be spatially and temporally intermittent and are stretched lengthwise along the main direction(s) of movement. Lanes often occur in multi-directional flows, but can also occur in different contexts.

\section{Model (general)}

1. A simplified representation or description of reality.

2. A model is always a simplification of reality that focuses on certain aspects. The range of validity or applicability is an essential part of any model. Thus, a model is always an approximation that has limited applicability. Conflicting models can coexist, e.g. the wave and particle models of light. 


\section{Model (acceleration-based)}

In pedestrian dynamics, an acceleration-based model is a model that describes the motion of pedestrians using a system of second order differential equations (possibly depending also on the pedestrians inner state).

Note: Social force models are examples of acceleration-based models. In these models, social interactions are supposed to modify the acceleration of pedestrians and thus are expressed using "social forces".

\section{Model (agent-based)}

A model in which individual autonomous (potentially heterogeneous) entities that have their own attribute values and may interact with each other and the environment according to a set of rules are represented. Interactions between agents in such models can lead to emergent behaviours.

\section{Model (cellular automata)}

1. A discrete model, where the next state of one cell is determined by both rules and the state of its neighbouring cells

2. In a cellular automaton model all relevant variables (space, time, state) are discrete and not continuous.

\section{Model (computational)}

1. The translation of a theoretical/conceptual model into an executable computer program.

2. A computational model is a mathematical model that uses computational resources to study the behavior of a system via computer simulations.

\section{Model (conceptual)}

A collection of variables, rules, parameters and relationships that captures an abstract representation of reality.

\section{Model (deterministic)}

A model that uses mathematical expressions to produce the same result each time the method is used with the same set of input data values.

\section{Model (empirical)}

A model based on observable real-world data (observable can be descriptions, interview, experimental data, case studies). 


\section{Model (formal)}

1. A model in which a system is expressed via clearly defined variables and relationships between variables.

2. These models tend to be expressed according to logical or mathematical rules and can be tested formally (automatically, mathematically).

\section{Models (hybrid)}

A combination of at least two computational models that differ in their approach, scale, time, space, environment, movement or other crowd entity characteristics.

\section{Models (macroscopic)}

Models that do not distinguish individuals. The system dynamics are described using aggregate quantities, such as densities or flows. Examples include approaches based on fluid dynamics and hand calculation methods.

\section{Models (machine learning)}

Models that consist of functions learnt from data that map input data to an output.

\section{Models (mesoscopic)}

1. Set in between microscopic and macroscopic models. It does not aim to describe aspects, such as the motion and behaviour, of each individual, but only certain aspects.

2. A model which considers agents as individuals, but describes their behaviour as aggregated relationships.

3. Model for a particle system describing the evolution of the probability distribution of particles in the phase space (often position-and-velocity space).

\section{Models (microscopic)}

Model of a particle system in which the dynamics of each particle are addressed individually (i.e. through a dedicated set of equations and/or algorithms). Examples include cellular automata and acceleration-based models.

\section{Models (micro-, meso-, and macroscopic)}

Micro-/Meso-/Macroscopic-models are labels for models that specify on what level behaviour is described/determined. E.g. micro = individuals determine the emergent collective behaviour, meso = individuals describe their behaviour through aggregated relationships, macro $=$ behaviours are produced through aggregated descriptions. 


\section{Models (multi-scale)}

A model which adopts different modelling scales to represent variables or aspects of the system.

\section{Model (probabilistic or stochastic)}

Model that describes the system dynamics in terms of probability distributions. This concept is not equivalent to the concept of noise added to models. However, adding noise to a deterministic model converts it into a stochastic model.

\section{Model (velocity-based)}

In pedestrian dynamics, a velocity-based model is a model that assigns in (typically discrete) time steps the velocity of pedestrians based on the current state (e.g. pedestrian velocity, position, other pedestrians' velocities and positions, etc.). This approach is often adopted in agent-based models. The basic assumption of these models is that the inertia of pedestrians is negligible with respect to the relevant time scale, i.e. pedestrians can easily reach their desired velocity in a time step. Such models may be derived from acceleration-based models by considering the limit when the relaxation term tends to zero.

\section{Navigation mesh}

Subdivision of the navigable space into polygonal regions enriched by a dual graph representing the connectivity between the regions.

\section{Network}

Structure connecting entities through relations. In psychology entities are typically persons or collections of people. In mathematics networks are generally modeled via graphs (collections of nodes and edges).

\section{Others}

Those who are not oneself, recognizing that oneself can be defined at an individual or group level.

\section{Pedestrian}

1. Person moving on foot in a publicly accessible area. Subcategories include: pedestriancommuter, pedestrian-shopper, pedestrian-traveller.

2. Person moving on foot in a publicly accessible area (phases of waiting or pausing included).

Open questions: when pedestrians stop moving, are they still pedestrians? When they sit, are they still a pedestrian? Does motivation/goal have an effect. Is it a matter of moving between spaces or moving within spaces? 


\section{Phenomenon (collective)}

A specific "event" in the dynamics of a crowd which is to be observed, described, explained, simulated, modelled, etc.

\section{Pitch invasion}

Audience deliberately entering a sports pitch as an illegitimate act of occupying the space to which they are not entitled. For example, in 1977 Scottish fans entered the pitch of Wembley stadium after their national football team had defeated the English team.

\section{Queuing}

Collective recognition (social norm) that the order of arrival determines the order in which people are being processed. For example, in a queue overtaking may be forbidden even if empty spaces would allow for it. This collective recognition is manifested in different psychological and physical ways.

\section{Rituals (collective)}

Collective rituals are group activities where there is a recognized set of behavioural patterns that is repeated through time. Social/group identity is both an input and output of participation on collective rituals.

\section{Routing}

Strategic planning from point of origin to final destination (e.g. planning a route from home to work). Related to wayfinding, but typically a longer journey than wayfinding.

\section{Social appraisal}

Assessment of the meaning of events (social situations) to the self and the ability of the self to deal with these events. The self can be defined at the individual or the group level. For example, "is this an emergency? Can this emergency be dealt with?" With an understanding that the self can be defined at the individual or group level.

\section{Social contagion, Social transfer, Social spread}

1. Non-automatic transfer of emotions, behaviours, information or cognition based on parameters concerning sender, receiver and channel. 
2. An alternative definition of contagion suggests that spread is automatic. The origin of the term is to spread merely through touch. It also comes with the baggage of "classical" crowd psychology which infers crowd irrationality, susceptibility and passivity. Contemporary crowd psychologists argue that we should abandon the language of "contagion" in favour of influence/spread.

\section{Social identity}

Social identity is defining an entity in terms of its membership of a social group along with the meaning that membership has for the entity.

\section{Social norm}

Shared definition of appropriate conduct, setting limits on behaviour (injunctive norm); what people tend to do (descriptive norm). Can apply to the appropriate ways to think (cognition), feel (emotion) and act (behaviour)

\section{Social relation}

Individual's representation of their social position relative to others. Individuals represent their social relation to others on different levels, i.e. group level and individual level. For example, individuals may move closer to or further away from others depending on their representation. It is important not to over-define and leave the definition somewhat open. Sub-definitions are necessary when moving to a specific application. It is important not to presuppose personal or group relations in this definition.

\section{Trajectory}

1. Continuous function from time to space resulting in a curve connecting an origin to a destination.

2. A trajectory is the path of an object moving through space as a function of time. In experimental data on pedestrian dynamics this is often captured via the path of the head. For models in pedestrian dynamics, it is typically an idealised location of pedestrians that does not consider body movements that arise from pedestrians shifting their weight from one foot onto the other (swaying). For fused data the trajectory can be enriched with additional data of the object (e.g. gaze direction).

\section{Wayfinding}

Wayfinding is the process of individuals taking cues from the environment to find their way as they progress through a journey from an origin to a destination. For example, an individual may move from the table to the door that they can see, and then from that door to the end of the corridor that they can see. Wayfinding is related to routing, but typically involves shorter journeys. 


\section{Discussion}

As stated above, we do not view this glossary as a collection of finalized and formal definitions, but as a starting point for a discussion on more consistent use of terminology in research on crowd dynamics. Some of the terms listed above have also been defined elsewhere in the literature and comparison to these additional definitions is highly recommended.

Whilst preparing this glossary, several additional suggestions that may be helpful to research in the field were made. These included the suggestion of additional terms (see below), as well as the suggestion to create a "blacklist" of misleading terms that are susceptible to misuse and may create misunderstandings (e.g. mob, mob mentality, herding, (mass) panic, contagion, stampede).

For completeness, we include a list of terms that were suggested, but for which no definitions were provided yet (in alphabetical order):

- Character

- Collective

- Collective dynamics

- Context and setting

- Crowd disaster

- Dispersion, mean-square displacement, diffusion?

- Egress

- Event

- Features of material environment

- Gathering

- Hydrodynamic relation

- Incident

- Levels of groups

- Mass

- Mass communication

- Mega-sport events

- Model (risk-based)

- Model (rule-based, decision-based) 
- National/historical events

- Nudging devices

- Occupant

- Person, people

- Protests

- Social movements

- Social ontology analyses

- Solidary behaviour

- Spread

- Very large groups

- Walking behaviour

Acknowledgements The workshop attendees gratefully acknowledge the support of the Lorentz Center in Leiden, The Netherlands, for the workshop Physics and Psychology of Human Crowd Dynamics (5 Nov - 9 Nov 2018). The workshop organizers acknowledge the financial support received by the Lorentz Center and by the Netherlands Organisation for Scientific Research (NWO). 